\title{
COMPOSITION OF HAAR PARAPRODUCTS: THE RANDOM CASE
}

\author{
DMITRIY BILYK, MICHAEL T. LACEY*, XIAOCHUN LI ${ }^{\dagger}$, AND BRETT D. WICK
}

\section{Definitions and Main Theorems}

We phrase the (difficult) open question which motivates the consideration of this paper. Let $\mathcal{D}$ be the dyadic grid, and $\left\{h_{I}: I \in \mathcal{D}\right\}$ the $L^{2}$ normalized Haar basis, namely

$$
h_{I}=|I|^{-1 / 2}\left(-\mathbf{1}_{I_{\text {left }}}+\mathbf{1}_{I_{\text {right }}}\right)
$$

We also use the notation $h_{I}^{0}=h_{I}$, indicating that the Haar function $h_{I}$ has integral zero. Set $h_{I}^{1} \stackrel{\text { def }}{=}\left|h_{I}^{0}\right|$, the subscript ${ }^{1}$ indicating that $h_{I}^{1}$ has non-zero integral.

A (classical dyadic) paraproduct with symbol $b$ is one of the operators

$$
\mathrm{B}(b, f)=\sum_{I \in \mathcal{D}} \frac{\left\langle b, h_{I}\right\rangle}{\sqrt{|I|}}\left\langle f, h_{I}^{\epsilon}\right\rangle h_{I}^{\delta} .
$$

Here, $\epsilon, \delta \in\{0,1\}$, with one of the two being zero and the other one. Then, it is well known that the operator $\mathrm{B}(b, \cdot)$ is bounded iff the symbol $b$ is in dyadic BMO. In particular, the following equivalence is a standard part of the literature, and essentially a direct consequence of the Carleson Embedding Theorem.

$$
\|\mathrm{B}(b, \cdot)\|_{2 \rightarrow 2} \simeq \sup _{J \in \mathcal{D}}\left[|J|^{-1} \sum_{I \subset J}\left\langle b, h_{I}\right\rangle^{2}\right]^{1 / 2} .
$$

An outstanding question concerns the composition of paraproducts: Namely, if one considers two paraproducts, each of which is potentially unbounded, can one meaningfully characterize when the composition is a bounded operator on $L^{2}$ ?

Characterize Compositions of Paraproducts that are Bounded. Let $\mathrm{B}(b, \cdot)$ and $\mathrm{B}\left(b^{\prime}, \cdot\right)$ be two possibly unbounded paraproducts. Provide a (non-trivial) characterization of the quantity

$$
\left\|\mathrm{B}\left(b, \mathrm{~B}\left(b^{\prime}, \cdot\right)\right)\right\|_{2 \rightarrow 2}
$$

\footnotetext{
*. Research supported in part by a National Science Foundation Grant.

$\dagger$. Research supported in part by a National Science Foundation Grant.

$\ddagger$. Research supported in part by a National Science Foundation Grant and the Fields Institute.
} 
(There are distinct versions of this question, depending upon how the paraproducts are defined.)

We answer a substantially easier variant of this question, in which the sum over the dyadic intervals is suitably randomized.

Motivations for this question arise from formulations of several closely related questions. (1) Which compositions of Toeplitz operators are bounded? (Axler et al., 1978; Zheng, 1996; Stroethoff and Zheng, 2002). (2) Which compositions of Hankel operators are bounded? (Vol'berg and Ivanov, 1987; Zheng, 1996) (3) For which pairs of weights $(u, v)$ is the Hilbert transform bounded as a map from $L^{2}(d u)$ to $L^{2}(d v)$ ? The question posed above is a discrete and combinatorial version of each of these questions. For prior work on it, or closely related questions, see (Pott and Smith, 2004). Each of these questions pose interesting and significant variants, which we leave to the interested reader to pursue in the citations we have provided. The work of Nazarov, Treil and Volberg on (3), in papers that are both together and separate, are highly recommended. See (Nazarov et al., 1999; Nazarov and Volberg, 2002; Nazarov and Trell', 1996; , 1996; Volberg, 2003). The paper (Blasco and Pott, 2005) studies the role of randomized paraproducts in the theory of product $B M O$.

There is one variant of these questions that has been completely solved. Eric Sawyer (Sawyer, 1982) characterized the two weight problem for the Maximal Function. Also see (Sawyer, 1982b; , 1983).

We turn to the main results of this paper. Fix a sequence of constants $\mathbf{b}=\left\{b_{I}: I \in \mathcal{D}\right\}$. For choices of $\epsilon, \delta \in\{0,1\}$, set

$$
\mathrm{P}_{\mathbf{b}}^{\epsilon, \delta}(f) \stackrel{\text { def }}{=} \sum_{I \in \mathcal{D}} b_{I} h_{I}^{\epsilon} \otimes h_{I}^{\delta}
$$

There are some comments in order. First, the typical choice of $\mathbf{b}$ is $\left\{\left\langle b, h_{I}\right\rangle / \sqrt{|I|}: I \in \mathcal{D}\right\}$, where $b$ is a $B M O$ function. There is however no function theory in our situation, which is one reason for taking $\mathbf{b}$ a numerical sequence. Another reason is that a second prominent example, arising from weighted inequalities is $\mathbf{b}=\left\{\left\langle b, h_{I}^{1}\right\rangle / \sqrt{|I|}: I \in \mathcal{D}\right\}$. We have chosen a normalization of $\mathbf{b}$ to make it a 'dimensionless' quantity.

It will be important for us that one of our paraproducts be permitted to vary over a class of paraproducts. This class is

$$
\mathrm{P}_{\mathbf{b}}^{\sigma, \epsilon, \delta}(f) \stackrel{\text { def }}{=} \sum_{I \in \mathcal{D}} \sigma_{I} b_{I} h_{I}^{\epsilon} \otimes h_{I}^{\delta} \quad \text { where } \quad \sigma=\left\{\sigma_{I}: I \in \mathcal{D}\right\} \in\{-1,+1\}^{\mathcal{D}} .
$$

Our primary results concern the case where the $\sigma_{I}$ are independent, identically, uniformly distributed, and where the estimates are uniform over the choices of signs. 
If a paraproduct does not have a ' 1 ' appearing in its exponents, it is very easy to estimate it's norm:

$$
\left\|\mathrm{P}_{\mathbf{b}}^{0,0}\right\|_{2} \simeq \sup _{I}\left|b_{I}\right|
$$

The critical case, and the one of interest to us, is when a ' 1 ' appears. Let us recall the classical result on the boundedness of these paraproducts.

1.3. Theorem. We have the equivalence of norms

$$
\left\|\mathrm{P}_{\mathbf{b}}^{0,1}\right\|_{2}=\left\|\mathrm{P}_{\mathbf{b}}^{1,0}\right\|_{2} \simeq \sup _{J \in \mathcal{D}}\left[|J|^{-1} \sum_{I \subset J} b_{I}^{2}|I|\right]^{1 / 2}
$$

This is the instance of the Carleson Embedding Theorem.

$$
\begin{aligned}
& \sum_{I \in \mathcal{D}} b_{I}^{2}\left\langle f, h_{I}^{1}\right\rangle^{2} \lesssim\left\|\left\{b_{I}\right\}\right\|_{\text {Carleson }}^{2}\|f\|_{2}^{2} \\
& \left\|\left\{b_{I}\right\}\right\|_{\text {Carleson }} \stackrel{\text { def }}{=} \sup _{J \in \mathcal{D}}\left[|J|^{-1} \sum_{I \subset J} b_{I}^{2}|I|\right]^{1 / 2} .
\end{aligned}
$$

We remark that paraproducts with two 1's arise in the setting of commutators with fractional integrals. But that is not our theme in this paper.

Our main interest is in the composition of paraproducts, with at least one of the two paraproducts having a 1 . The interest here is in finding necessary and sufficient conditions for the composition to be bounded, permitting the individual paraproducts to be unbounded. We concentrate on the random sign case, establishing necessary and sufficient conditions which are 'natural' extensions of the classical result above.

It is useful to set notation

$$
\|\mathrm{T}\|_{\mathbb{E}, p \rightarrow p} \stackrel{\text { def }}{=} \sup _{\|f\|_{p}=1} \mathbb{E}\|\mathrm{T} f\|_{p}
$$

With this definition, it is not necessarily the case $\|\mathrm{T}\|_{\mathbb{E}, p \rightarrow p} \neq\left\|\mathrm{T}^{*}\right\|_{\mathbb{E}, p^{\prime} \rightarrow p^{\prime}}$ for conjugate index $p^{\prime}$.

1.7. Theorem. We have the following equivalences, in which $\sigma$ denotes random choices of signs.

$$
\begin{aligned}
&\left\|\mathrm{P}_{\mathbf{b}}^{1,0} \mathrm{P}_{\boldsymbol{\beta}}^{\sigma, 0,1}\right\|_{\mathbb{E}, 2 \rightarrow 2} \simeq\left\|\left\{b_{I} \cdot \beta_{I}\right\}\right\|_{\text {Carleson }} \\
&\left\|\mathrm{P}_{\mathbf{b}}^{0,1} \mathrm{P}_{\boldsymbol{\beta}}^{\sigma, 0,0}\right\|_{\mathbb{E}, 2 \rightarrow 2} \simeq \sup _{J}\left[\frac{\beta_{J}^{2}}{|J|} \sum_{I: I \subsetneq J} b_{I}^{2} \mid I\right]^{1 / 2} \\
&\left\|\mathrm{P}_{\mathbf{b}}^{0,0} \mathrm{P}_{\boldsymbol{\beta}}^{\sigma, 1,0}\right\|_{\mathbb{E}, 2 \rightarrow 2} \simeq \sup _{J}\left[\frac{\beta_{J}^{2}}{|J|} \sum_{I: J \subsetneq I} b_{I}^{2}|I|\right]^{1 / 2}
\end{aligned}
$$




$$
\begin{aligned}
& \left\|\mathrm{P}_{\mathbf{b}}^{0,1} \mathrm{P}_{\boldsymbol{\beta}}^{\sigma, 0,1}\right\|_{\mathbb{E}, 2 \rightarrow 2} \simeq\left\|\left[\frac{\beta_{J}^{2}}{|J|} \sum_{I: I \subsetneq J} b_{I}^{2}|I|\right]^{1 / 2}\right\|_{\text {Carleson }}, \\
& \left\|\mathrm{P}_{\mathbf{b}}^{0,1} \mathrm{P}_{\boldsymbol{\beta}}^{\sigma, 1,0}\right\|_{\mathbb{E}, 2 \rightarrow 2} \simeq\left\{\left\|\mathrm{P}_{\mathbf{b}}^{\sigma, 0,1} \mathrm{P}_{\boldsymbol{\beta}}^{0,0}\right\|_{\mathbb{E}, 2 \rightarrow 2}+\left\|\mathrm{P}_{\mathbf{b}}^{0,0} \mathrm{P}_{\boldsymbol{\beta}}^{0,0}\right\|_{2 \rightarrow 2}+\left\|\mathrm{P}_{\boldsymbol{\beta}}^{0,0} \mathrm{P}_{\mathbf{b}}^{\sigma, 1,0}\right\|_{\mathbb{E}, 2 \rightarrow 2}\right\}
\end{aligned}
$$

In the first equivalence (1.8) is elementary in nature and we include it only for the sake of completeness. This case dramatically simplifies as it is highly local: there are no interactions between dyadic scales which are widely separated in the the hyperbolic metric.

The second line, (1.9) is a characterization in same terms as those of Carleson measures. The essential difference is that the non-local: The coefficients $b_{I}$ and $\beta_{J}$ must be paired even when $I$ and $J$ are widely separated in scale. In this line, it is essential that we have the randomization fall on the paraproducts as described above. Without this term, the right hand side is certainly not sufficient for the boundedness of the paraproduct.

Note that for any choices of signs $\sigma$ and $\widetilde{\sigma}$, and any $\phi \in L^{2}$, we have

$$
\left\|\mathrm{P}_{\mathbf{b}}^{\sigma, 0,1} \mathrm{P}_{\boldsymbol{\beta}}^{0,0} \phi\right\|_{2}=\left\|\mathrm{P}_{\mathbf{b}}^{\widetilde{\sigma}, 0,1} \mathrm{P}_{\boldsymbol{\beta}}^{0,0} \phi\right\|_{2}
$$

That is, in (1.9) we need not consider the other randomization $\left\|\mathrm{P}_{\mathbf{b}}^{\sigma, 0,1} \mathrm{P}_{\boldsymbol{\beta}}^{0,0}\right\|_{\mathbb{E}, 2 \rightarrow 2}$.

A similar set of comments apply to (1.10). In particular, the characterization here is also of a non-local nature.

Note that also $\mathrm{P}_{\mathbf{b}}^{0,0} \mathrm{P}_{\boldsymbol{\beta}}^{0,0}$ trivially diagonalizes, we we easily have

$$
\left\|\mathrm{P}_{\mathbf{b}}^{0,0} \mathrm{P}_{\boldsymbol{\beta}}^{0,0}\right\|_{2 \rightarrow 2}=\sup _{I}\left|b_{I} \beta_{I}\right|
$$

In view of (1.9), the right hand sides of (1.12) can be replaced with an explicit equivalence for the norm in the same spirit as the Carleson measure condition. Again, these conditions are certainly not sufficient for the case when one does not average over choices of signs.

There is another instance that arise naturally, through a connection with two weight inequalities. Let

$$
\mathrm{T}_{\sigma}=\sum_{I \in \mathcal{D}} \sigma_{I} h_{I} \otimes h_{I}
$$

by an random Haar multiplier sequence, with $\sigma_{I} \in\{ \pm 1\}$. Let $\mathrm{M}_{b} \varphi \stackrel{\text { def }}{=} b \cdot \varphi$. 
1.13. Theorem. Let $b, \beta$ be functions, with finitely supported Haar expansion. We have the equivalence of norms

$$
\begin{aligned}
\left\|\mathrm{M}_{b} \mathrm{~T}_{\sigma} \mathrm{M}_{\beta}\right\|_{\mathbb{E}, 2 \rightarrow 2} & \simeq \sup _{I} \mathbb{E}\left\{\left\|\mathrm{M}_{b} \mathrm{~T}_{\sigma} \mathrm{M}_{\beta} h_{I}^{1}\right\|_{2}+\left\|\mathrm{M}_{\beta} \mathrm{T}_{\sigma} \mathrm{M}_{\beta} h_{I}^{1}\right\|_{2}\right\} \\
\simeq \sup _{I} & \frac{\left|\left\langle b, h_{I}^{1}\right\rangle\right|}{|I|}\left[\int_{I} \beta^{2} d x\right]^{1 / 2}+\frac{\left|\left\langle\beta, h_{I}^{1}\right\rangle\right|}{|I|}\left[\int_{I} b^{2} d x\right]^{1 / 2} \\
& +\left\|\frac{\left\langle b, h_{J}\right\rangle}{|J|}\left[\int_{J} \beta^{2} d x\right]^{1 / 2}\right\|_{\text {Carleson }} \\
& +\left\|\frac{\left\langle\beta, h_{J}\right\rangle}{|J|}\left[\int_{J} b^{2} d x\right]^{1 / 2}\right\|_{\text {Carleson }} .
\end{aligned}
$$

Acknowledgements. We would like to express our sincere gratitude to the Shanks Foundation at Vanderbilt University. Their generosity allowed the authors to meet for a weekend at Vanderbilt University to discuss aspects of this question. Special thanks are due to the organizers of the Thematic Program in Harmonic Analysis at the Fields Institute in WinterSpring of 2008, as well as the staff of the Institute. It is during that semester that the paper took its final form.

\section{Proof of Theorem 1.7}

The principal way that random signs enters into the the arguments is through averaging of all possible signs. Namely, if $\sigma_{J}$ are independent identically distributed random variables, taking the values \pm 1 with equal probability, we have

$$
\mathbb{E}\left\|\sum_{j} \sigma_{J} g_{j}\right\|_{2}^{2}=\sum_{j}\left\|g_{j}\right\|_{2}^{2} .
$$

That is we gain additional orthogonality by averaging over signs.

To use the random choices of signs on the paraproduct, we need random choices of signs indexed by the dyadic intervals $\left\{\sigma_{I}: I \in \mathcal{D}\right\}$, and we write

$$
\mathrm{P}_{\mathbf{b}}^{\sigma, \epsilon, \delta} \stackrel{\text { def }}{=} \sum_{I} \sigma_{I} b_{I} h_{I}^{\epsilon} \otimes h_{I}^{\delta}
$$

Proof of (1.8). Observe that the presence of the two inside 0's diagonalizes the operator.

$$
\mathrm{Q}=\mathrm{P}^{\sigma, 1,0} \mathrm{P}^{0,1}=\sum_{I} \sigma_{I} \cdot b_{I} \beta_{I} \cdot h_{I}^{1} \otimes h_{I}^{1} .
$$


That is, the composition is a paraproduct with two 1's, and symbol is the product symbol. Clearly, random choices of signs are essential. We have

$$
\mathbb{E}\|\mathrm{Q} \phi\|_{2}^{2}=\sum_{I \in \mathcal{D}}\left|b_{I} \beta_{I}\right|^{2}\left\langle\phi, h_{I}^{1}\right\rangle^{2}
$$

And so we can appeal to (1.5) to conclude this case.

Proof of (1.9). Observe that

$$
\mathrm{Q}=\mathrm{P}_{\mathbf{b}}^{0,1} \mathrm{P}_{\boldsymbol{\beta}}^{\sigma, 0,0}=\sum_{\substack{I, J \in \mathcal{D} \\ I \subsetneq J}} \sigma_{J} \tau_{I, J} \cdot b_{I} \beta_{J} \cdot \frac{\sqrt{|I|}}{\sqrt{|J|}} h_{I} \otimes h_{J}
$$

where $\tau_{I, J}$ is the sign of $h_{J}$ on the interval $I$. Since $I$ is strictly contained in the interval $J$, this is well defined.

Apply $\mathrm{Q}$ to $h_{J}$ to see that

$$
\begin{aligned}
\left\|\mathrm{Q} h_{J}\right\|_{2}^{2} & =\left\|\sum_{I \subsetneq J} \sigma_{J} \tau_{I, J} \cdot b_{I} \beta_{J} \cdot \frac{\sqrt{|I|}}{\sqrt{|J|}} h_{I}\right\|_{2}^{2} \\
& =\sum_{I \subsetneq J}\left|b_{I} \beta_{J}\right|^{2} \frac{|I|}{|J|}
\end{aligned}
$$

The operator norm dominates the supremum over $J$ on the right, which proves the lower bound on the operator norm.

Let us assume that

$$
\sup _{J} \sum_{I \subsetneq J}\left|b_{I} \beta_{J}\right|^{2} \frac{|I|}{|J|} \leq 1
$$

and establish an absolute upper bound on the the operator Q. Apply Q to function $\phi$ of $L^{2}$ norm one.

$$
\begin{aligned}
\mathbb{E}\|\mathrm{Q} \phi\|_{2}^{2} & =\mathbb{E}\left\|\sum_{\substack{I, J \in \mathcal{D} \\
I \subsetneq J}} \sigma_{J} \tau_{I, J} \cdot b_{I} \beta_{J} \cdot \frac{\sqrt{|I|}}{\sqrt{|J|}}\left\langle\phi, h_{J}\right\rangle h_{I}\right\|_{2}^{2} \\
& =\sum_{I} \mathbb{E}\left|\sum_{I \subsetneq J} \sigma_{J} \tau_{I, J} \cdot b_{I} \beta_{J} \cdot \frac{\sqrt{|I|}}{\sqrt{|J|}}\left\langle\phi, h_{J}\right\rangle\right|^{2} \\
& =\sum_{\substack{I, J \in \mathcal{D} \\
I \subsetneq J}}\left(b_{I} \beta_{J}\right)^{2} \frac{|I|}{|J|}\left\langle\phi, h_{J}\right\rangle^{2} \\
& \leq\|\phi\|_{2}^{2} \sup _{J} \sum_{I \subsetneq J}\left|b_{I} \beta_{J}\right|^{2} \frac{|I|}{|J|} .
\end{aligned}
$$

This completes our proof. 
Proof of (1.10). As in the proof of (1.9), the proof of the lower bound on the operator norm does not depend upon the averaging over signs. Apply $\mathrm{Q}=\mathrm{P}_{\mathbf{b}}^{0,0} \mathrm{P}_{\boldsymbol{\beta}}^{\sigma, 1,0}$ to $h_{J}$ to see that

$$
\begin{aligned}
\left\|\mathrm{Q} h_{J}\right\|_{2}^{2} & =\left\|\sum_{I: J \subsetneq I} \sigma_{J} \tau_{J, I} \cdot b_{I} \beta_{J} \cdot \frac{\sqrt{|I|}}{\sqrt{|J|}} h_{I}\right\|_{2}^{2} \\
& =\sum_{I: J \subsetneq I}\left|b_{I} \beta_{J}\right|^{2} \frac{|I|}{|J|} .
\end{aligned}
$$

Note that the operator norm clearly dominates the supremum over $J$ on the right, and this proves the first lower bound on the operator norm.

Now let us assume that

$$
\sup _{J} \sum_{I: J \subsetneq I}\left|b_{I} \beta_{J}\right|^{2} \frac{|I|}{|J|}=1,
$$

and let us find an absolute upper bound on operator Q defined in (2.1). Apply Q to a function $f$ of $L^{2}$ norm one.

$$
\begin{aligned}
\mathbb{E}\|\mathrm{Q} f\|_{2}^{2} & =\mathbb{E}\left\|\sum_{\substack{I, J \\
J \subsetneq I}} \sigma_{J} \tau_{I, J} \cdot b_{I} \beta_{J} \cdot \frac{\sqrt{|I|}}{\sqrt{|J|}}\left\langle f, h_{J}\right\rangle h_{I}\right\|_{2}^{2} \\
& =\sum_{I} \mathbb{E}\left|\sum_{J: J \subsetneq I} \sigma_{J} \tau_{I, J} \cdot b_{I} \beta_{J} \cdot \frac{\sqrt{|I|}}{\sqrt{|J|}}\left\langle f, h_{J}\right\rangle\right|^{2} \\
& =\sum_{\substack{I, J \\
J \subsetneq I}}\left|b_{I} \beta_{J}\right|^{2} \frac{\mid I J}{|J|}\left\langle f, h_{J}\right\rangle^{2} \\
& \leq\|f\|_{2}^{2} \sup _{J} \sum_{I: J \subsetneq I}\left|b_{I} \beta_{J}\right|^{2} \frac{|I|}{|J|} .
\end{aligned}
$$

This completes the proof of the boundedness of this Q.

Proof of (1.11). In this proof, we calculate

$$
\begin{aligned}
\mathbb{E}\left\|\mathrm{P}_{\mathbf{b}}^{0,1} \mathrm{P}_{\boldsymbol{\beta}}^{\sigma, 0,1} \phi\right\|_{2}^{2} & =\sum_{I} b_{I}^{2}|I| \mathbb{E}\left|\sum_{J: I \subsetneq J} \sigma_{J} \tau_{I, J} \frac{\beta_{J}}{\sqrt{|J|}}\left\langle\phi, h_{J}^{1}\right\rangle\right|^{2} \\
& =\sum_{J}\left\langle\phi, h_{J}^{1}\right\rangle^{2} \cdot \frac{\beta_{J}^{2}}{|J|} \sum_{I: I \subsetneq J} b_{I}^{2}|I| .
\end{aligned}
$$

It is then clear that we have

$$
\left\|\mathrm{P}_{\mathbf{b}}^{0,1} \mathrm{P}_{\boldsymbol{\beta}}^{\sigma, 0,1}\right\|_{\mathbb{E}, 2 \rightarrow 2} \simeq\left\|\frac{\beta_{J}}{\sqrt{|J|}}\left[\sum_{I: I \subsetneq J J} b_{I}^{2}|I|\right]^{1 / 2}\right\|_{\text {Carleson }}
$$


This argument does not shed light on the case when the random choices of signs are imposed on the other paraproduct. Namely, we obviously have

$$
\begin{aligned}
\left\|\mathrm{P}_{\mathbf{b}}^{\sigma, 0,1} \mathrm{P}_{\boldsymbol{\beta}}^{0,1} \phi\right\|_{2}^{2} & =\left\|\mathrm{P}_{\mathbf{b}}^{0,1} \mathrm{P}_{\boldsymbol{\beta}}^{0,1} \phi\right\|_{2}^{2} \\
& =\sum_{I} b_{I}^{2}\left\langle h_{I}^{1}, \mathrm{P}_{\boldsymbol{\beta}}^{0,1} \phi\right\rangle^{2} .
\end{aligned}
$$

That is, the randomization of signs plays no role if placed in this coordinate.

Proof of (1.12). Observe that

$$
\begin{aligned}
\mathbb{E}\left\|\mathrm{P}_{\mathbf{b}}^{0,1} \mathrm{P}_{\boldsymbol{\beta}}^{\sigma, 1,0} \phi\right\|_{2}^{2} & =\sum_{I} b_{I}^{2} \mathbb{E}\left|\sum_{I \cap J \neq \emptyset} \sigma_{J}\left\langle h_{I}^{1}, h_{J}^{1}\right\rangle \beta_{J}\left\langle\phi, h_{J}\right\rangle\right|^{2} \\
& =\sum_{J}\left\langle\phi, h_{J}\right\rangle^{2} \beta_{J}^{2} \sum_{I: I \cap J \neq \emptyset} b_{I}^{2}\left\langle h_{I}^{1}, h_{J}^{1}\right\rangle^{2} b_{I}^{2} .
\end{aligned}
$$

Therefore, we have

$$
\begin{aligned}
\left\|\mathrm{P}_{\mathbf{b}}^{0,1} \mathrm{P}_{\boldsymbol{\beta}}^{\sigma, 1,0}\right\|_{\mathbb{E}, 2 \rightarrow 2}^{2} & \simeq \sup _{J} \beta_{J}^{2} \sum_{I: I \cap J \neq \emptyset} b_{I}^{2}\left\langle h_{I}^{1}, h_{J}^{1}\right\rangle^{2} b_{I}^{2} \\
& \simeq \sup _{J} \frac{\beta_{J}^{2}}{|J|} \sum_{I: I \subsetneq J} b_{I}^{2}|I|+\beta_{J}^{2} b_{J}^{2}+\beta_{J}^{2}|J| \sum_{I: J \subsetneq I} \frac{b_{I}^{2}}{|I|} .
\end{aligned}
$$

By the previous part of the proof, this last supremum, times an absolute constant, dominates the sum of the operator norms

$$
\left\|\mathrm{P}_{\mathbf{b}}^{0,1} \mathrm{P}_{\boldsymbol{\beta}}^{\sigma, 0,0}\right\|_{\mathbb{E}, 2 \rightarrow 2}^{2}+\left\|\mathrm{P}_{\mathbf{b}}^{0,0} \mathrm{P}_{\boldsymbol{\beta}}^{\sigma, 0,0}\right\|_{\mathbb{E}, 2 \rightarrow 2}^{2}+\left\|\mathrm{P}_{\boldsymbol{\beta}}^{0,1} \mathrm{P}_{\mathbf{b}}^{\sigma, 0,0}\right\|_{\mathbb{E}, 2 \rightarrow 2}^{2}
$$

Conversely, by appropriate selection of test function, this last term also dominates the supremum, so our proof is complete.

\section{Random Two Weight: Sufficient Direction}

We consider the upper bound of the operator norms in (1.14) by the expressions involving the Carleson measure norm. To do so, we expand the composition $\mathrm{M}_{b} \mathrm{TM}_{\beta}$ as a sum of paraproducts, as considered in the first half of the paper.

Define a paraproduct operator by

$$
\mathrm{P}_{b, \alpha}^{\beta, \gamma} \stackrel{\text { def }}{=} \sum_{I \in \mathcal{D}} \frac{\left\langle b, h_{I}^{\alpha}\right\rangle}{\sqrt{|I|}} h_{I}^{\beta} \otimes h_{I}^{\gamma}
$$


Here, $\alpha, \beta, \gamma \in\{0,1\}$. Observe that we have the usual expansion

$$
\begin{aligned}
\mathrm{M}_{b} \varphi= & \left\{\sum_{I}\left\langle b, h_{I}\right\rangle h_{I}\right\}\left\{\sum_{I}\left\langle\varphi, h_{I}\right\rangle h_{I}\right\} \\
= & \sum_{I} \frac{\left\langle b, h_{I}\right\rangle}{\sqrt{|I|}}\left\langle\varphi, h_{I}\right\rangle h_{I}^{1} \\
& +\sum_{I} \frac{\left\langle b, h_{I}\right\rangle}{\sqrt{|I|}}\left\langle\varphi, h_{I}^{1}\right\rangle h_{I}^{1} \\
& +\sum_{I} \frac{\left\langle b, h_{I}^{1}\right\rangle}{\sqrt{|I|}}\left\langle\varphi, h_{I}\right\rangle h_{I} \\
= & \mathrm{P}_{b, 0}^{0,1} \varphi+\mathrm{P}_{b, 0}^{1,0} \varphi+\mathrm{P}_{b, 1}^{0,0} \varphi
\end{aligned}
$$

There are nine cases in the expansion of the operator $\mathrm{M}_{b} \mathrm{TM}_{\beta}$. We examine them in turn. The symbols associated to the paraproducts reduce to one of four possibilities. For the function $b$ they are

$$
\mathbf{b}^{0} \stackrel{\text { def }}{=}\left\{\left\langle b, h_{I}^{0}\right\rangle / \sqrt{|I|}\right\}, \quad \mathbf{b}^{1} \stackrel{\text { def }}{=}\left\{\left\langle b, h_{I}^{1}\right\rangle / \sqrt{|I|}\right\} .
$$

For the function $\beta$ we have the same two possibilities, for which we use the notation $\boldsymbol{\beta}^{0}$ and $\boldsymbol{\beta}^{1}$.

It is essential to note that if we are forming a composition with paraproducts with a 0 in the interior of the composition, then the random Haar multiplier can be imposed on that zero, resulting in a composition of random paraproducts. Namely,

$$
\mathrm{P}_{b, \epsilon_{1}}^{\epsilon_{2}, 0} \mathrm{TP}_{\beta, \delta_{1}}^{\delta_{2}, \delta_{3}}=\mathrm{P}_{\mathbf{b}^{\epsilon_{1}}}^{\sigma, \epsilon_{2}, 0} \mathrm{P}_{\boldsymbol{\beta}^{\delta_{1}}}^{\delta_{2}, \delta_{3}}
$$

Here, we are using notations from the first half of the paper.

We discuss the nine separate cases that arise from the expansion of

$$
\mathrm{M}_{b} \mathrm{~T} \mathrm{M}_{\beta}=\left\{\mathrm{P}_{b, 1}^{0,0}+\mathrm{P}_{b, 0}^{1,0}+\mathrm{P}_{b, 0}^{0,1}\right\} \mathrm{T}\left\{\mathrm{P}_{\beta, 1}^{0,0}+\mathrm{P}_{\beta, 0}^{1,0}+\mathrm{P}_{\beta, 0}^{0,1}\right\}
$$

In each case, we can describe a necessary and sufficient condition for the boundedness of that individual term. These terms together provide a sufficient condition for the boundedness of the composition. Indeed, the different conditions are listed in (3.2) to (3.10) and altogether, they are dominated by the the right hand side of (1.14).

Case of $00_{1}-01_{0}$ and $10_{0}-00_{1}$. The paraproduct is

$$
\mathrm{P}_{b, 1}^{0,0} \mathrm{TP}_{\beta, 0}^{0,1}=\mathrm{P}_{\mathbf{b}^{1}}^{0,0} \mathrm{P}_{\boldsymbol{\beta}^{0}}^{\sigma, 0,1}
$$


This is now a classical paraproduct, and one can estimate it's norm as

$$
\left\|\mathrm{P}_{b, 1}^{0,0} \mathrm{TP}_{\beta, 0}^{0,1}\right\|_{2 \rightarrow 2} \simeq\left\|\frac{\left\langle b, h_{I}^{1}\right\rangle}{\sqrt{|I|}} \cdot \frac{\left\langle\beta, h_{I}\right\rangle}{\sqrt{|I|}}\right\|_{\text {Carleson }}
$$

By duality, we see that

$$
\left\|\mathrm{P}_{b, 0}^{1,0} \mathrm{TP}_{\beta, 1}^{0,0}\right\|_{2 \rightarrow 2} \simeq\left\|\frac{\left\langle b, h_{I}\right\rangle}{\sqrt{|I|}} \cdot \frac{\left\langle\beta, h_{I}^{1}\right\rangle}{\sqrt{|I|}}\right\|_{\text {Carleson }}
$$

Case of $00_{1}-00_{1}$. The paraproduct is

$$
\mathrm{P}_{b, 1}^{0,0} \mathrm{~T} \mathrm{P}_{\beta, 1}^{0,0}=\mathrm{P}_{\mathbf{b}^{1}}^{\sigma, 0} \mathrm{P}_{\boldsymbol{\beta}^{1}}^{0,0}
$$

This is a composition with all zeros, which immediately diagonalizes to give

$$
\left\|\mathrm{P}_{b, 1}^{0,0} \mathrm{TP}_{\beta, 1}^{0,0}\right\|_{2 \rightarrow 2}=\sup _{I} \frac{\left|\left\langle b, h_{I}^{1}\right\rangle\left\langle\beta, h_{I}^{1}\right\rangle\right|}{|I|}
$$

Case of $00_{1}-10_{0}$ and $01_{0}-00_{1}$. The paraproduct in the case of $00_{1}-10_{0}$ is

$$
\mathrm{P}_{b, 1}^{0,0} \mathrm{TP}_{\beta, 0}^{1,0}=\mathrm{P}_{\mathbf{b}^{1}}^{\sigma, 0,0} \mathrm{P}_{\boldsymbol{\beta}^{0}}^{1,0}
$$

This is a paraproduct of the type considered in (1.9) (with the roles of $b$ and $\beta$ reversed.) Thus, we have

$$
\begin{aligned}
\left\|\mathrm{P}_{b, 1}^{0,0} \mathrm{TP}_{\beta, 0}^{1,0}\right\|_{2 \rightarrow 2}^{2} & \simeq \sup _{J} \frac{\left\langle b, h_{J}^{1}\right\rangle^{2}}{|J|^{2}} \sum_{I \subsetneq J}\left\langle\beta, h_{I}\right\rangle^{2} \\
& \simeq \sup _{I}\left\langle\beta, h_{I}\right\rangle^{2} \sum_{I \subsetneq J} \frac{\left\langle b, h_{J}^{1}\right\rangle^{2}}{|J|^{2}}
\end{aligned}
$$

By duality, in the case of $01_{0}-00_{1}$ we have

$$
\begin{aligned}
\left\|\mathrm{P}_{b, 0}^{0,1} \mathrm{TP}_{\beta, 1}^{0,0}\right\|_{2 \rightarrow 2}^{2} & \simeq \sup _{J} \frac{\left\langle\beta, h_{J}^{1}\right\rangle^{2}}{|J|^{2}} \sum_{I \subset J}\left\langle b, h_{I}\right\rangle^{2} \\
& \simeq \sup _{I}\left\langle b, h_{I}\right\rangle^{2} \sum_{I \subsetneq J} \frac{\left\langle\beta, h_{J}^{1}\right\rangle^{2}}{|J|^{2}}
\end{aligned}
$$


Case of $01_{0}-10_{0}$. This case does not appeal to the first half of our paper, as the random Haar multiplier cannot be absorbed into either paraproduct. We estimate

$$
\begin{aligned}
\mathbb{E}\left\|\mathrm{P}_{b, 0}^{0,1} \mathrm{~T}_{\beta, 0}^{1,0} \phi\right\|_{2}^{2} & =\sum_{I} \frac{\left\langle b, h_{I}\right\rangle^{2}}{|I|} \mathbb{E}\left\langle h_{I}^{1}, \mathrm{TP}_{\beta, 0}^{1,0} \phi\right\rangle^{2} \\
& =\sum_{I}\left\langle b, h_{I}\right\rangle^{2} \sum_{J: I \subsetneq J} \frac{\left\langle\beta, h_{J}\right\rangle^{2}}{|J|}\left\langle\phi, h_{J}\right\rangle^{2}
\end{aligned}
$$

It is then clear that we have

$$
\left\|\mathrm{P}_{b, 0}^{0,1} \mathrm{TP}_{\beta, 0}^{1,0}\right\|_{2 \rightarrow 2}^{2} \simeq \sup _{J} \frac{\left\langle\beta, h_{J}\right\rangle^{2}}{|J|} \sum_{I: I \subsetneq J}\left\langle b, h_{I}\right\rangle^{2}
$$

The Case of $01_{0}-01_{0}$ and $10_{0}-10_{0}$. The case of $01_{0}-01_{0}$ is

$$
\mathrm{P}_{b, 0}^{0,1} \mathrm{TP}_{\beta, 0}^{0,1}=\mathrm{P}_{\mathbf{b}^{0}}^{0,1} \mathrm{P}_{\boldsymbol{\beta}^{0}}^{\sigma, 0,1} \text {. }
$$

Thus, we can appeal to (1.11) to conclude that

$$
\left\|\mathrm{P}_{b, 0}^{0,1} \mathrm{TP}_{\beta, 0}^{0,1}\right\|_{2} \simeq\left\|\frac{\left\langle\beta, h_{J}\right\rangle}{|J|}\left[\sum_{I: I \subsetneq J}\left\langle b, h_{I}\right\rangle^{2}\right]^{1 / 2}\right\|_{\text {Carleson }} .
$$

The case of $10_{0}-10_{0}$ is dual, and yields

$$
\left\|\mathrm{P}_{b, 0}^{1,0} \mathrm{TP}_{\beta, 0}^{1,0}\right\|_{2}^{2} \simeq\left\|\frac{\left\langle b, h_{J}\right\rangle}{|J|}\left[\sum_{I \subsetneq J}\left\langle\beta, h_{I}\right\rangle^{2}\right]^{1 / 2}\right\|_{\text {Carleson }} .
$$

The Case of $10_{0}-01_{0}$. This case reduces to that of (1.8).

$$
\begin{aligned}
\mathrm{P}_{b, 0}^{1,0} \mathrm{TP}_{\beta, 0}^{0,1} & =\mathrm{P}_{\mathbf{b}^{0}}^{\sigma, 1,0} \mathrm{P}_{\boldsymbol{\beta}^{0}}^{0,1} . \\
& \simeq\left\|\frac{\left\langle b, h_{I}\right\rangle\left\langle\beta, h_{I}\right\rangle}{|I|}\right\|_{\text {Carleson }} .
\end{aligned}
$$

\section{Random Two Weight: The Necessary Direction}

We show that the operator norms on the left in (1.14) provide an upper bound on the expressions involving Carleson measures.

Let us begin by applying the operators $\mathrm{M}_{b} \mathrm{~T} \mathrm{M}_{\beta}$ to the functions $h_{J}^{1}$. We have

$$
\begin{aligned}
\mathbb{E}\left\|\mathrm{M}_{b} \mathrm{TM}_{\beta}\right\|_{2 \rightarrow 2}^{2} & \geq \mathbb{E}\left\|\mathrm{M}_{b} \mathrm{TM}_{\beta} h_{J}^{1}\right\|_{2}^{2} \\
& \geq \frac{1}{|J|} \sum_{I \subset J}\left\|b \cdot h_{I} \cdot\left\langle\beta, h_{I}\right\rangle\right\|_{2}^{2}
\end{aligned}
$$




$$
\begin{aligned}
& =\frac{1}{|J|} \sum_{I \subset J} \frac{\left\langle\beta, h_{I}\right\rangle^{2}}{|I|} \int_{I} b^{2} d x \\
& =\frac{1}{|J|} \sum_{I \subset J} \frac{\left\langle\beta, h_{I}\right\rangle^{2}}{|I|}\left\{\left\langle b, h_{J}^{1}\right\rangle^{2}+\sum_{K \subsetneq J}\left\langle b, h_{J}\right\rangle^{2}\right\}
\end{aligned}
$$

This inequality, and the dual assertion, proves that the operator norm bounds the two terms involving the Carleson measure on the right in (1.14).

It remains to observe that we have

$$
\begin{aligned}
\mathbb{E}\left\|\mathrm{M}_{b} \mathrm{TM}_{\beta}\right\|_{2 \rightarrow 2} & \geq \mathbb{E}\left\|\mathrm{M}_{b} \mathrm{~T} \mathrm{M}_{\beta} h_{J}^{0}\right\|_{2}^{2} \\
& \geq \frac{\int_{J} b^{2} d x\left|\left\langle\beta, h_{J}^{0}\right\rangle\right|}{|J|^{2}}
\end{aligned}
$$

This, and the dual inequality, completes the proof of the operator bound bounding the the right hand side of (1.14).

\section{REFERENCES}

Axler, Sheldon, Sun-Yung A. Chang, and Donald Sarason. 1978. Products of Toeplitz operators, Integral Equations Operator Theory 1, no. 3, 285-309.MR511973 (80d:47039)

Blasco, Óscar and Sandra Pott. 2005. Dyadic BMO on the bidisk, Rev. Mat. Iberoamericana 21, no. 2, 483-510. MR 2174914 (2006h:42041)

Nazarov, F. L. and S. R. Trell'. 1996. The hunt for a Bellman function: applications to estimates for singular integral operators and to other classical problems of harmonic analysis, Algebra i Analiz 8, no. 5, 32-162 (Russian, with Russian summary); English transl.,. 1996, St. Petersburg Math. J. 8, no. 5, 721-824. MR 1428988 (99d:42026)

Nazarov, F., S. Treil, and A. Volberg. 1999. The Bellman functions and two-weight inequalities for Haar multipliers, J. Amer. Math. Soc. 12, no. 4, 909-928.MR1685781 (2000k:42009)

Nazarov, F. and A. Volberg. 2002. The Bellman function, the two-weight Hilbert transform, and embeddings of the model spaces $K_{\theta}$, J. Anal. Math. 87, 385-414. Dedicated to the memory of Thomas H. Wolff. MR 1945290 (2003j : 30081)

Pott, Sandra and Martin P. Smith. 2004. Paraproducts and Hankel operators of Schatten class via p-JohnNirenberg theorem, J. Funct. Anal. 217, no. 1, 38-78.MR2097606

Stroethoff, Karel and Dechao Zheng. 2002. Invertible Toeplitz products, J. Funct. Anal. 195, no. 1, 4870.MR1934352 (2003g:47051)

Sawyer, Eric T. 1983. Norm inequalities relating singular integrals and the maximal function, Studia Math. 75, no. 3, 253-263. MR 722250 (85c:42018)

. 1982a. A characterization of a two-weight norm inequality for maximal operators, Studia Math. 75 , no. 1, 1-11. MR 676801 (84i : 42032)

. 1982b. Two weight norm inequalities for certain maximal and integral operators, Harmonic analysis (Minneapolis, Minn., 1981), Lecture Notes in Math., vol. 908, Springer, Berlin, pp. 102-127. MR 654182 (83k:42020b)

Tachizawa, K. 2001. On weighted dyadic Carleson's inequalities, J. Inequal. Appl. 6, no. 4, 415433.MR1888434 (2003a:42027) 
Volberg, Alexander. 2003. Calderón-Zygmund capacities and operators on nonhomogeneous spaces, CBMS Regional Conference Series in Mathematics, vol. 100, Published for the Conference Board of the Mathematical Sciences, Washington, DC. MR 2019058 (2005c:42015)

Vol'berg, A. L. and O. V. Ivanov. 1987. Membership of the product of two Hankel operators in the Schattenvon Neumann class, Dokl. Akad. Nauk Ukrain. SSR Ser. A, no. 4, 3-6 (Russian, with English summary).MR898320 (88h:47037)

Zheng, Dechao. 1996. The distribution function inequality and products of Toeplitz operators and Hankel operators, J. Funct. Anal. 138, no. 2, 477-501.MR1395967 (97e:47040)

Dmitriy Bilyk, School of Mathematics, Georgia Institute of Technology, Atlanta GA 30332 ,

E-mail address: bilyk@math.gatech.edu

Michael T. Lacey, School of Mathematics, Georgia Institute of Technology, Atlanta GA 30332,

E-mail address: lacey@math.gatech.edu

Xiaochun li, Department of Mathematics, University of Illinois, Urbana-Champaign, UrBANA IL 61801,

E-mail address: xcli@math.uiuc.edu

Brett D. Wick, Current: Fields Institute, University of Toronto, Toronto, Ontario M5T 3J1 Canada., Permanent: University of South Carolina, Department of Mathematics, LeConte College, 1523 Greene Street, Columbia, SC 29208

E-mail address: wick@math.sc.edu 